\title{
Médiévales
}

Langues, Textes, Histoire

51 | automne 2006

L'Occident sur ses marges ( $\left.\mathrm{VI} \mathrm{e}^{\mathrm{e}} \mathrm{X}\right|^{\mathrm{e}}$ siècles)

\section{Cambrai-Magdebourg : les reliques des saints et l'intégration de la Lotharingie dans le royaume de Germanie au milieu du $\mathrm{x}^{\mathrm{e}}$ siècle}

Cambrai-Magdebourg: The Relics of the Saints and the Integration of Lotharingia into the Kingdom of Germany in the Middle of the 10th Century

\section{Paul Bertrand et Charles Mériaux}

\section{OpenEdition}

Journals

Édition électronique

URL : https://journals.openedition.org/medievales/1514

DOI : 10.4000/medievales. 1514

ISSN : $1777-5892$

Éditeur

Presses universitaires de Vincennes

Édition imprimée

Date de publication : 1 décembre 2006

Pagination : 85-96

ISBN : 978-2-84292-193-4

ISSN : 0751-2708

\section{Référence électronique}

Paul Bertrand et Charles Mériaux, «Cambrai-Magdebourg : les reliques des saints et l'intégration de la Lotharingie dans le royaume de Germanie au milieu du xe siècle », Médiévales [En ligne], 51 | automne 2006, mis en ligne le 03 novembre 2010, consulté le 23 avril 2022. URL : http://

journals.openedition.org/medievales/1514; DOI : https://doi.org/10.4000/medievales.1514

Ce document a été généré automatiquement le 23 avril 2022

Tous droits réservés 


\title{
Cambrai-Magdebourg : les reliques des saints et l'intégration de la Lotharingie dans le royaume de Germanie au milieu du $\mathrm{x}^{\mathrm{e}}$ siècle
}

\author{
Cambrai-Magdebourg: The Relics of the Saints and the Integration of \\ Lotharingia into the Kingdom of Germany in the Middle of the 10th Century
}

Paul Bertrand et Charles Mériaux

Pendant le haut Moyen Âge, l'échange de reliques obéissait à des préoccupations qui dépassaient le plan spirituel pour renforcer des relations sociales, familiales et politiques ${ }^{1}$. Hedwig Röckelein a ainsi récemment rappelé l'importance fondamentale qu'avaient eue les translations de reliques vers la Saxe au $\mathrm{Ix}^{\mathrm{e}}$ siècle : venues de Rome, d'Italie ou du cœur de la Francie, les reliques des saints contribuèrent puissamment à la christianisation de la Saxe et à l'intégration rapide de son aristocratie dans la société franque $^{2}$. Au siècle suivant, la situation a toutefois entièrement changé. La royauté et l'aristocratie du royaume de Germanie revendiquaient au même titre que celles du royaume occidental l'héritage politique et culturel carolingien et, dans la première moitié du xe siècle, Henri I ${ }^{\text {er }} l^{\prime}$ Oiseleur $(919 \nmid 936)$ puis son fils Otton $I^{\text {er }}(936 † 973)$ firent de leur duché de Saxe le cœur d'une nouvelle renaissance carolingienne qui culmina le 2 février 962 par le couronnement impérial d'Otton à Rome reçu des mains du pape Jean XII. Comme l'a très justement écrit Michel Bur, la Saxe est au cœur de l'histoire de l'Europe au $\mathrm{x}^{\mathrm{e}}$ siècle : « désormais c'est à la France ou à la Bourgogne de s'intégrer à une Europe dominée par les Saxons». Or dans cette intégration progressive - Michel Bur parle aussi de "satellisation ${ }^{3}{ }^{-}$- d'une partie de l'Occident dans l'espace politique saxon, les reliques des saints jouèrent une nouvelle fois un rôle de premier plan. Il n'est naturellement pas question de présenter ici l'ensemble des dévotions que privilégièrent les premiers souverains ottoniens ainsi que les aristocrates laïcs et ecclésiastiques qui 
leur étaient liés, dont certains ont d'ailleurs été déjà bien étudiés comme Thierry de Metz $(965 \dagger 984)^{4}$.

Nous voudrions nous attarder ici sur un dossier de taille plus modeste, celui des translations de reliques du diocèse de Cambrai vers la Saxe au milieu du $\mathrm{x}^{\mathrm{e}}$ siècle. Il conviendra dans un premier temps de présenter rapidement la documentation liturgique dont dispose aujourd'hui encore l'historien pour appréhender ces translations. Plus que la documentation narrative, c'est elle qui permet de repérer l'introduction de nouveaux cultes, en particulier à Magdebourg. On aura ensuite à cœur de dégager les implications politiques de ces cérémonies religieuses.

\section{La documentation liturgique de Magdebourg}

3 C'est pour l'Église de Magdebourg, fondée en 937 et érigée en archevêché en 968, que la documentation liturgique est la plus abondante ${ }^{5}$. On conserve en effet de nos jours un sacramentaire en usage au sein du chapitre cathédral sous l'épiscopat de Tagino (1004-1012). Ce codex avait été composé dans la deuxième moitié du $\mathrm{Ix}^{\mathrm{e}}$ siècle à SaintGall, vraisemblablement pour la cathédrale de Ratisbonne. Après un passage relativement bref à Magdebourg, le manuscrit s'est retrouvé à l'abbaye de Stavelot dans le courant $\mathrm{du} \mathrm{XI} \mathrm{XI}^{\mathrm{e}}$ siècle ; il est aujourd'hui conservé à Bruxelles ${ }^{6}$. À Magdebourg, son calendrier a été complété (les fêtes des saints spécialement honorés sur place sont marquées d'un $\mathrm{M}$ ) et des notices nécrologiques ont été introduites. Un calendrier très similaire, utilisé à Mayence au $\mathrm{IX}^{\mathrm{e}}$ siècle puis adapté aux usages de Magdebourg à $l^{\prime}$ extrême fin du $x^{e}$ siècle ou au début du siècle suivant, est aussi conservé à Rome ${ }^{7}$. Les deux calendriers semblent indépendants l'un de l'autre. Une liste de reliques a aussi été conservée dans la documentation diplomatique (donation de reliques de Magdebourg par l'archevêque Wichmann aux cisterciennes d'Ichterhsausen, en Thuringe, en $1166^{8}$ ). Une étude plus exhaustive que la nôtre pourrait enfin s'appuyer sur les innombrables documents liturgiques de l'extrême fin du Moyen Âge et de l'époque moderne.

\section{Les saints Géry et Aubert à Magdebourg}

4 Depuis l'époque carolingienne, deux saints évêques de Cambrai, Géry (Gaugericus) et Aubert (Autbertus), sont communément honorés dans l'ensemble du monde franc et les grands martyrologes carolingiens mentionnent tous le jour de leur fête, respectivement les 11 août et 13 décembre. Il ne fait cependant aucun doute qu'ils faisaient l'objet d'un culte particulier à Magdebourg autour de l'an mil car leurs deux noms sont signalés par un $\mathrm{M}$ dans les deux calendriers aujourd'hui conservés et, au XII ${ }^{\mathrm{e}}$ sì̀cle, des reliques de Géry étaient mentionnées dans le trésor de la cathédrale puisqu'une partie d'entre elles furent cédées aux religieuses d'Ichtershausen. L'explication de cette dévotion particulière aux saints évêques cambrésiens est fournie par l'auteur de la Vie d'Aubert, qui est de toute évidence un chanoine de la cathédrale de Cambrai écrivant entre 1023-1024 ${ }^{9}$. Celui-ci rapporte longuement la demande faite par Otton $\mathrm{I}^{\mathrm{er}}$ à l'évêque Fulbert de Cambrai (933/934 † 956) de lui adresser les reliques des saints Géry et Aubert pour la cathédrale de Magdebourg (ce qui constitue un léger - et excusable nous semble-t-il - anachronisme puisque Saint-Maurice, fondé en 937, ne fut érigé en chapitre métropolitain qu'en 968). Pris entre l'« amour du souverain » et « la crainte de 
l'offense divine " (car il était impensable de priver Cambrai de ses patrons célestes), Fulbert se résolut à céder des parcelles du corps d'Aubert accompagnées des restes de deux défunts remarquables: l'évêque Thierry $(\dagger$ 863) et un prêtre du diocèse. Ces reliques voyagèrent "sous le faux nom" des saints Géry et Aubert ${ }^{10}$. L'événement‘fu910〉 peut être en partie daté puisqu'il eut lieu après la donation par Otton de l'abbaye Saint-Géry à l'évêque, le 30 avril 948, qui libérait opportunément Fulbert de la potentia consularis - entendons la domination du comte sur une partie de la cité $^{11}$. Tout cela explique assez l'embarras de l'évêque. On montrera plus loin que les circonstances de cette translation se comprennent très bien à la lumière des relations particulières nouées entre la cour et l'évêque Fulbert à la fin des années 940. La réalité de cette translation ne doit sans doute pas être soupçonnée.

\section{Madelberte, Maxellende et Saturnine}

Seconde abbesse de Maubeuge à la fin du vII ${ }^{\mathrm{e}}$ siècle, Madelberte (Madalberta) était une figure de dévotion locale en Hainaut. Son culte est attesté à Maubeuge à partir du $\mathrm{x}^{\mathrm{e}}$ siècle ${ }^{12}$. Mais il est particulièrement curieux de le voir aussi signalé à Magdebourg dès la fin de ce siècle. Madelberte est mentionnée au 7 septembre dans les deux calendriers. Ses reliques sont citées dans la liste de l'archevêque Wichmann au XII ${ }^{\mathrm{e}}$ siècle. Elles semblent même avoir acquis une importance particulière dans le courant du Moyen Âge puisqu'elles étaient déposées, avec celles de saint Maurice, sur le sarcophage

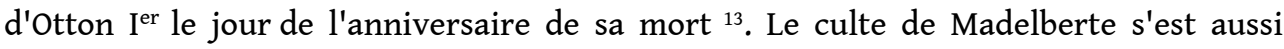
diffusé hors de Magdebourg. Ses reliques furent en effet introduites dans l'autel SaintDenis de la cathédrale d'Halberstadt lors de la grande cérémonie de consécration du sanctuaire le 18 octobre $992^{14}$.

Sainte Maxellende (Maxellendis) est connue par une Vie rédigée avant le début $\mathrm{du} \mathrm{XI}^{\mathrm{e}}$ siècle car l'auteur des Gesta des évêques de Cambrai, composés en 1024/1025, la connaît et la résume librement. Il n'existe pas de témoignage antérieur du culte de cette jeune fille originaire de Caudry, à quelques kilomètres à l'est de Cambrai. À la fin du $\mathrm{VII}^{\mathrm{e}}$ siècle, promise en mariage à ses parents, elle se serait fermement dérobée. Son fiancé finit par l'enlever. Devant sa résistance obstinée, il la tua. D'abord enseveli dans la basilique du domaine voisin de Pomeriolas, son corps fut rapporté à Caudry trois ans plus tard et une communauté religieuse aurait été fondée autour de son tombeau. On sait qu'au $\mathrm{x}^{\mathrm{e}}$ siècle ses reliques furent transportées dans l'Église Saint-Martin située près des murs de Cambrai ${ }^{15}$. En 1025, ses reliques furent confiées à l'abbaye SaintAndré du Cateau-Cambrésis nouvellement fondée par l'évêque Gérard ${ }^{\text {er }}$. Maxellende est donc une sainte dont le culte apparaît assez tardivement dans la documentation et de manière très localisée autour de Cambrai.

Il y a une exception à cela. La depositio de Maxellende (13 novembre) a en effet été introduite dans deux calendriers en usage à l'abbaye royale d'Essen sur la Ruhr au même moment que celle de saint Florin de Ramosch dans l'Engadine (17 novembre) ${ }^{16}$. Les spécialistes reconnaissent dans les deux cas la main qui officiait principalement dans le scriptorium avant l'incendie de l'établissement au milieu des années 940. On retrouve aussi le nom de Maxellende dans le calendrier d'un sacramentaire utilisé à Essen à une époque plus avancée dans le $\mathrm{x}^{\mathrm{e}}$ siècle ${ }^{17}$. La documentation postérieure montre que la sainte n'a guère été mise à l'honneur par les religieuses (pour lesquelles elle représentait toutefois un modèle tout à fait adapté), éclipsée sans doute par la 
renommée de saints plus prestigieux : Cosme et Damien, Quentin, Mars, Walburge et Florin et bien d'autres encore ${ }^{18}$. Toutefois la présence de Maxellende dans les calendriers du $\mathrm{x}^{\mathrm{e}}$ siècle pourrait signifier que des reliques de la sainte - ou au moins de très modestes fragments - ont pu être cédées à Essen. Naturellement les circonstances précises d'une éventuelle translation nous échappent totalement. Subsiste donc cette seule évidence : Maxellende, sainte locale du Cambrésis, était bien honorée à Essen à partir des années 940, c'est-à-dire sous l'épiscopat de Fulbert de Cambrai (quand ses reliques étaient déjà très vraisemblablement conservées à Saint-Martin, hors des murs de la cité), mais, semble-t-il, quelques années avant l'arrivée des reliques des saints Géry et Aubert à Magdebourg. Il est imprudent d'imaginer que les deux translations aient été menées exactement au même moment.

En 1024/1025, le rédacteur des Gesta des évêques de Cambrai donne une liste commentée des établissements religieux des diocèses d'Arras et Cambrai. Il rappelle ainsi que, non loin de Cambrai, à Sanctis (aujourd'hui Sains-lès-Marquion), avait existé dans le passé une communauté féminine (basilica puellarum) rassemblée autour des reliques d'une sainte Saturnine (Saturnina). Ce qu'il rapporte à son sujet est assez confus. À une époque non précisée, cette jeune fille originaire de Germanie s'était consacrée à Dieu, mais ses parents l'avaient fiancée contre son gré. Elle quitta le domicile familial et parvint dans les environs de Sains. Son fiancé la rattrapa et la décapita. Prenant sa tête dans ses mains, elle eut le temps de gagner l'église du lieu avant de mourir. Un monastère féminin aurait été fondé. Plus tard des Saxons de passage à Sains seraient venus emporter une partie de ses reliques ${ }^{19}$. Le martyre de Saturnine est visiblement inspiré de celui de sainte Maxellende dont il vient d'être question. La mention de la Germanie et des Saxons est en revanche intéressante car on retrouve en Saxe - à Neuenheerse, Paderborn et Corvey - des attestations du culte d'une sainte Saturnine. Mais les sources sont un peu plus tardives ( $\mathrm{XI}^{\mathrm{e}}$-XII ${ }^{\mathrm{e}}$ siècles) et font parfois de Saturnine une martyre romaine ${ }^{20}$. Il y a assurément là un écheveau obscur de traditions hagiographiques qui demanderait une étude plus fouillée ${ }^{21}$. On se contentera donc de relever le témoignage de l'auteur des Gesta, persuadé qu'à une époque mal précisée, des «Saxons » étaient venus emporter des reliques locales. Il est alors tentant de rapprocher ces traditions des translations en Saxe des saints Géry et Aubert ainsi que de celles, probables, des saintes Madelberte et Maxellende. Mais il faut savoir que la région a été parcourue dès le $\mathrm{Ix}^{\mathrm{e}}$ siècle par des voyageurs et des convois de reliques comme en témoignent la halte que les reliques de saint Liboire et des autres saints du Mans firent à la civitatula (ou castellum) de Bavay lors de leur translation vers Paderborn en mai $836^{22}$. Il n'est donc pas exclu qu'on ait conservé à Cambrai le souvenir d'un événement bien antérieur au $\mathrm{x}^{\mathrm{e}}$ siècle.

9 Il apparaît toutefois clairement que plusieurs saints cambrésiens ont été honorés en Saxe dès le $\mathrm{x}^{\mathrm{e}}$ siècle. Les circonstances de la translation des reliques des saints Géry et Aubert sous l'épiscopat de Fulbert, peu après 948, sont bien documentées. L'introduction du culte de sainte Maxellende à Essen fut légèrement plus précoce, dans la première moitié des années 940 , donc déjà sous l'épiscopat de Fulbert. En ce qui concerne Madelberte, les sources attestant son culte en Saxe sont un peu plus tardives, mais, étant donné le rayonnement très local de ce culte en Hainaut, on pourrait raisonnablement se demander si l'introduction de son culte à Magdebourg ne s'est pas faite dans le sillage de la translation des saints Géry et Aubert. Quant à Saturnine, en l'état actuel des connaissances, il est plus difficile de la rattacher en toute certitude à ce 
mouvement. En somme, même s'il convient de rester prudent, plusieurs indices plaident pour une introduction concertée de plusieurs cultes cambrésiens en Saxe sous l'épiscopat de Fulbert. Il convient donc désormais de proposer quelques explications à ce phénomène.

\section{La signification de ces translations}

10 Il ne faudrait pas exagérer l'importance du culte des saints cambrésiens à Magdebourg. En 937, la fondation de Saint-Maurice (dédié aux saints Innocents dans un premier temps), un an après l'avènement d'Otton $\mathrm{I}^{\mathrm{er}}$, est le point de départ d'une politique de contrôle de la frontière orientale de la Saxe, au-delà de l'Elbe, mais également d'évangélisation en direction des populations slaves. Otton ambitionnait de faire de Magdebourg le centre de cet ample mouvement, d'ériger rapidement la ville en siège d'une province métropolitaine. En 955 puis à nouveau en 962, les résistances de l'archevêque de Mayence et de l'évêque voisin de Halberstadt, conjuguées aux hésitations de la papauté, expliquent que ce projet n'ait finalement été mené à bien qu'en 968. Adalbert, issu de la chancellerie royale, en fut le premier titulaire. Cinq ans plus tard, Otton Ir manifestait tout l'attachement qu'il avait pour Saint-Maurice, qu'il avait abondamment doté et enrichi, en choisissant de se faire inhumer dans la cathédrale ${ }^{23}$. Comme l'a écrit Walter Schlesinger, Otton entendait bien faire de Magdebourg l'« Aix-la-Chapelle de l'Est ${ }^{24}$.

11 Cet attachement est à rapprocher des efforts du souverain pour rassembler à SaintMaurice un prestigieux trésor de reliques au premier rang desquelles il faut citer celles de saints de la Légion thébaine, martyrisés à la fin du ${ }^{\mathrm{e}} \mathrm{e}^{\mathrm{e}}$ siècle et honorés au monastère d'Agaune ${ }^{25}$. À Noël 960, eut lieu la translation solennelle de reliques de saint Maurice venues de Ratisbonne ${ }^{26}$. Ce lot sera par la suite complété par une donation, faite en 1004 par Henri II, de reliques du même martyr puisées dans le trésor de la chapelle royale ${ }^{27}$. On sait également qu'Otton $I^{\text {er }}$ - qui passa plus de dix années de sa vie en Italie - chargea son chapelain Dodon de faire venir de la péninsule de nombreux corps saints à Magdebourg ${ }^{28}$. Il faut enfin savoir que Magdebourg fut ensuite au centre d'un vaste mouvement de redistribution de reliques vers les évêchés et les monastères saxons qui, par ailleurs, ont pu aussi disposer de leurs propres réseaux d'approvisionnement ${ }^{29}$. Il apparaît donc clairement que les Ottoniens ont eu à cœur d'assurer à Magdebourg et à la Saxe toute entière les meilleures protections célestes, celles de saints martyrs romains, en attendant de proposer au tournant de l'an mil, des modèles dignes de vénération au sein de leur propre famille ${ }^{30}$. Les saints de Cambrai ne constituaient donc qu'un lot infime, et assurément pas le plus prestigieux. C'est bien pour cette raison qu'il faut essayer de mieux comprendre sa présence à Magdebourg au $\mathrm{x}^{\mathrm{e}}$ siècle.

En 843 , Cambrai fit partie du royaume de Lothaire ${ }^{\mathrm{er}}$ puis de son fils Lothaire II. La cité épiscopale suivit ensuite les destinées de cette partie de la Lotharingie, acquise par Charles le Chauve au traité de Meersen (870), puis absorbée définitivement en 925 dans le royaume de Germanie par Henri I ${ }^{\text {er }}$. Cela n'a pas empêché le duc Gislebert, suivi par une bonne partie de l'aristocratie locale, qui, à partir de 936, pouvaient compter sur le soutien du roi de Francie occidentale, de manifester la plus grande indépendance à l'égard d'Otton I ${ }^{\mathrm{er}}$. En 939 , la révolte avortée du duc et sa mort brutale renforcèrent momentanément la position du souverain saxon qui eut néanmoins à affronter très vite une opposition aristocratique bientôt emmenée (à partir de 944) par son futur beau- 
frère, le duc Conrad le Rouge ${ }^{31}$. Dans la deuxième moitié des années 940 , Otton s'est donc soucié de trouver des soutiens efficaces au sein de l'épiscopat lotharingien, une politique que poursuivit avec beaucoup de succès son frère Brunon à partir de $953^{32}$. À Cambrai, les résultats de cette politique furent rapidement concluants : en 948, on l'a dit, le souverain retira au comte l'abbaye Saint-Géry pour la confier à l'évêque désormais seul maitre de la cité et de son suburbium. Aux dires de l'auteur de la Vie d'Aubert, la translation des saints Géry et Aubert vers Magdebourg intervint dans ce contexte précis d'obligations contractées par l'évêque à l'égard du souverain saxon. On sait aussi que Fulbert joua un rôle important lors du concile d'Ingelheim (7 juin 948) en défendant les prétentions d'Artaud, candidat d'Otton et de Louis d'Outre-Mer, sur le siège métropolitain de Reims contre Hugues de Vermandois ${ }^{33}$. Définitivement installé à Reims, le même Artaud fit d'ailleurs parvenir des reliques des saints Apollinaire et Timothée, deux martyrs rémois, à Magdebourg ${ }^{34}$.

13 Si l'on accepte l'hypothèse que des reliques des saintes Madelberte et Maxellende furent envoyées en Saxe à peu près à la même époque - et avec l'accord du même Fulbert -, on peut également proposer une autre interprétation. De la même manière que la donation des reliques de saint Maurice en 960 (et plus encore sans doute la donation de la sainte Lance par Rodophe II à Henri I $^{\text {er }}$ dès 926) pouvait signifier la dépendance dans laquelle se plaçait le royaume de Bourgogne vis-à-vis de la Germanie, la translation d'un lot de reliques cambrésiennes manifestait aux yeux de tous l'intégration de la partie la plus occidentale de la Lotharingie (dont la frontière était fixée sur l'Escaut) dans l'espace politique ottonien. L'événement avait sans doute d'autant plus d'importance qu'il ne correspondait pas encore vraiment à un sentiment unanimement partagé : l'aristocratie lotharingienne était encore hésitante dans sa fidélité à Otton, et la famille carolingienne en Francie occidentale n'avait pas renoncé à s'emparer du duché. L'utilisation de translations de reliques pour garantir et manifester l'unité profonde d'un espace politique ou religieux n'est pas nouvelle. Qu'il suffise ici de citer, au siècle précédent, les demandes de corps saints faites à ses suffragants par le grand archevêque Hincmar de Reims $(845 \dagger 882)$ afin, dit Jean Devisse, de "symboliser l'unité de la province» ${ }^{35}$. Certes, il n'est pas possible de montrer que telle était la raison qui a poussé Otton à faire venir des reliques cambrésiennes en Saxe. En revanche, il est tout à fait intéressant de noter que cette interprétation est clairement exprimée dès le début $\mathrm{du} \mathrm{XI}^{\mathrm{e}}$ siècle par l'auteur de la Vie d'Aubert. Ce dernier rappelle en effet la nécessité qu'il y avait, un demi-siècle plus tôt, d'envoyer en Saxe des reliques pour assurer la défense spirituelle de Magdebourg contre les menaces païennes encore vivaces. Il déplore dans un premier temps la demande d'Otton car elle signifiait le départ des saints qui avaient jusque-là assuré la prospérité de la cité ${ }^{36}$. Mais, favorablement impressionné par l'habileté de Fulbert à répondre à la demande du roi sans priver complètement Cambrai de ses patrons célestes, il se réjouit finalement de voir que deux cités situées aux «confins» du royaume aient pu être protégées par les mêmes saints patrons ${ }^{37}$. L'auteur manifeste assurément une conscience très vive de l'unité de l'Empire, de son Église et de ses saints en ce début du XI ${ }^{\mathrm{e}}$ siècle.

14 La translation des reliques de saint Géry et de saint Aubert à Magdebourg peu après 948 est le seul événement de ce type entre Cambrai et la Saxe attesté à la fois par les sources narratives et liturgiques, mais il semble bien qu'ait existé au même moment un mouvement de translations d'une plus grande ampleur qui aurait aussi concerné les 
reliques de sainte Madelberte de Maubeuge (vers Magdebourg) et celles de sainte Maxellende de Caudry (vers Essen). Les traditions relatives à sainte Saturnine de Sainslès-Marquion sont plus confuses, mais pourraient être aussi interprétées dans ce contexte. Quelles en furent les causes? Ces translations révèlent assurément le souci

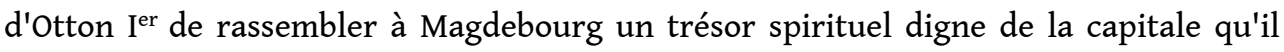
entendait fonder; elles s'enracinent dans la politique lotharingienne de ce même souverain après 944 et en particulier dans les relations étroites nouées avec l'évêque Fulbert de Cambrai ; elles manifestent enfin, sur un plan spirituel, l'intégration de la Lotharingie dans l'espace ottonien dont le cœur était saxon. Alors qu'au IX ${ }^{\mathrm{e}}$ siècle, les translations de reliques de la Francie vers la Saxe étaient le signe de l'intégration de cette dernière dans l'Empire carolingien, au siècle suivant, c'est exactement l'inverse : la périphérie est devenue centre et le centre périphérie.

\section{NOTES}

1. Commode état de la question dans E. BozóKY, "L'initiative et la participation du pouvoir laïc dans les translations de reliques au haut Moyen Âge. Esquisse typologique ", Sources. Travaux historiques, t. 51-52, 1997, p. 39-57 ; voir également les dossiers rassemblés dans U. SWINARSKI, Herrschen mit den Heiligen. Kirchenbesuche, Pilgerfahrten und Heiligenverehrung früh- und hochmittelalterlicher Herrscher (ca. 500-1200), Berne, Berlin, etc., 1996 (Geist und Werk der Zeiten, 78) ainsi que quelques références fondamentales: H. FichTENAU, «Zum Reliquienwesen im früheren Mittelalter », Mitteilungen des Instituts für österreichische Geschichtsforschung, t. 60, 1952, p. 60-89 ; N. HERRMANN-MASCARD, Les reliques des saints. Formation coutumière d'un droit, Paris, 1975 ; M. HeInZELmann, Translationsberichte und andere Quellen des Reliquienkultes, Turnhout, 1979 (Typologie des sources du Moyen Âge occidental, 33) ; P. GEARY, Le vol des reliques au Moyen Âge, Paris, 1993 ; Les reliques. Objets, cultes, symboles, dir. E. BozóKY et A.-M. HeLVÉTIUS, Turnhout, 1999 (Hagiologia, 1).

2. H. RöCKELEIN, Reliquientranslationen nach Sachsen im 9. Jahrhundert. Über Kommunikation und Öffentlichkeit im Frühmittelalter, Stuttgart, 2002 (Beihefte der Francia, 48).

3. M. BUR, «Adalbéron, archevêque de Reims, reconsidéré ", dans Le roi de France et son royaume autour de l'an mil, Paris, 1992, p. 55-63 ; réimp. dans ID., La Champagne médiévale. Recueil d'articles, Langres, 2005, p. 719-736 (citations p. 724 et 734).

4. E. DUPRÉ THESEIDER, « La "grande rapina dei corpi santi" dall'Italia al tempo di Ottone I », dans Festschrift Percy Ernst Schramm zu seinem siebzigsten Geburtstag von Schülern und Freunden zugeeignet, t. 1, Wiesbaden, 1964, p. 420-432; A. WAGNER, «Collection de reliques et pouvoir épiscopal au x siècle. L'exemple de l'évêque Thierry $I^{\mathrm{er}}$ de Metz ", dans Revue d'histoire de l'Église de France, t. 83, 1997, p. 317-341.

5. B. SCHWINEKÖPER et G. W ENTZ, Das Erzbistum Magdebourg, t.1-1, Das Domstift St. Moritz in Magdeburg, Berlin/New-York, 1972, spéc. p. 216-238; à compléter aussi par H. KÜHNE, Ostensio reliquiarum. Untersuchungen über Entstehung, Ausbreitung, Gestalt und Funktion der Heiltumsweisungen im römisch-deutschen Regnum, Berlin/New-York, 2000, p. 228-229. 
6. Bibl. royale, ms. 1814-1816 ; un fac-similé du calendrier-nécrologe ( $\mathrm{f}^{\circ} 1 \mathrm{r}^{\circ}-17 \mathrm{v}^{\circ}$ ) a été publié dans Die Totenbücher von Merseburg, Magdeburg und Lüneburg, G. ALTHOFF et J. W OLLASCH éd., Hanovre, 1983 (MGH Libri memoriales et necrologia, nova series 2), p. 35-68.

7. Bibl. Vaticane, Cod. Pal. Lat. $1447, \mathrm{f}^{\circ} 12 \mathrm{r}^{\circ}-17 \mathrm{v}^{\mathrm{o}}$; éd. F. Jostes, «Saxonica ", Zeitschrift für deutsches Altertum, t. 40, 1896, p. 148-159.

8. Urkundenbuch des Erzstifts Magdeburg, t. 1, 937-1192, F. ISRAËL et W. MöLLENBERG éd., Magdebourg, 1937, n 315A, p. 398-403 (Wichmann charge le chantre de la cathédrale, Siegfrid, de procéder à la donation après sa mort) et $\mathrm{n}^{\circ}$ 315B, p. 403-406 (donation dudit Siegfrid le 7 janvier 1166).

9. Vita Autberti, J. GHESQuière éd., Acta Sanctorum Belgii selecta, t. 3, Bruxelles, 1785, p. 538-565, ici IV, 30-32, p. 562-563; pour l'attribution de ce texte à un chanoine cambrésien (et non à l'évêque Fulbert de Chartres), voir C. MÉRIAUX, "L'auteur de la Vita Autberti», dans M. DiESENBERGER et M. NIEDERKORN-BRUCK dir., Hagiographische Überlieferung im Frühmittelalter zwischen Niederschrift und Wiederschrift, à paraître.

10. Nous empruntons cette expression à H. PlATELLE, «Reliques circulant sous un faux nom : formalisme et religion populaire », dans La Religion populaire, Paris, 1979, p. 95-102; 〈fe〈fo910〉voir également sur ce thème ID., « Erreur sur la personne. Contribution à l'histoire de l'imposture au Moyen Âge ", dans Universitas. Philosophie, Théologie, Lettres, Histoire, Questions d'aujourd'hui, no spécial des Mélanges de science religieuse pour le centenaire des Facultés catholiques de Lille, Lille, 1977, p. 117-145 ; réimp. dans ID., Présence de l'Au-delà. Une vision médiévale du monde, Villeneuved'Ascq, 2004, p. 29-50 (ici p. 39-40).

11. Le détail des événements (et des sources) est donné dans C. MÉRIAuX, «Fulbert, évêque d'Arras et de Cambrai $(933 / 934 † 956) »$, dans L'Église et la société entre Seine et Rhin (Ve-XVI siècle). Recueil d'études d'histoire du Moyen Âge en l'honneur de Bernard Delmaire, Revue du Nord, t.86, $\mathrm{n}^{\circ}$ 356-357, juillet-décembre 2004, p. 525-542, spéc. p. 526-529.

12. P. BERTRAND, «La Vie de sainte Madelberte de Maubeuge », Analecta Bollandiana, t. 115, 1997, p. 39-76; ID., "Sainte Madelberte à Soignies : aux origines d'un culte récent ", dans J. DEVESELEER dir., Reliques et châsses de la collégiale de Soignies. Objets, cultes et traditions, Soignies, 2001 (Les cahiers du chapitre, 8), p. 89-108.

13. Liber de consuetudinibus divinorum ecclesiae Magdeburgensis (composé au milieu du XIII siècle), G. SELlo éd., « Dom-Altertümer », Geschichtsblätter für Stadt und Land Magdeburg, t. 26, 1891, p. 137-138.

14. Annalista Saxo, Chronica, G. WAITZ éd., Monumenta Germania historica [désormais: MGH], Scriptores [désormais: SS], t. 6, Hanovre, 1844, p. 553-777, à la p. 637 (œuvre rédigée dans les années 1140, sans doute par Arnold, abbé de Berge près de Magdebourg); Gesta episcoporum Halberstadensium, L. WEILAND éd., MGH, SS, t. 23, Hanovre, 1880, p. 79-123, à la p. 87 (compilés au tout début $\mathrm{du}$ XIII ${ }^{\mathrm{e}}$ siècle); sur cette historiographie saxonne, voir en dernier lieu K. NASS, Die Reichskronik des Annalista Saxo und die sächsische Geschichtsschreibung im 12. Jahrhundert, Hanovre, 1996 (MGH, Schriften, 41).

15. Passio Maxellendis prima, H. Bevenot éd., Acta Sanctorum Belgii selecta, t. 3, Bruxelles, 1785, p. 580-587 (sur ce texte, voir L. VAN DER ESSEN, Étude critique et littéraire sur les vitae des saints mérovingiens de l'ancienne Belgique, Louvain/Paris, 1907 (Recueil de travaux de l'Université de Louvain, $1^{\text {re }}$ série, 17), p. 277-281) ; Gesta episcoporum Cameracensium, L. BETHMANN éd., MGH, SS, t. 7, Hanovre, 1846, p. 393-489, I, 24-25, p. 410 (sur l'œuvre, voir E. VAN M INGROoT, « Kritisch onderzoek omtrent de datering van de Gesta episcoporum Cameracensium », Revue belge de philologie et d'histoire, t. 53, 1975, p. 281-332).

16. Düsseldorf, Universitäts- und Landesbibliothek, D1, fo $222 \mathrm{r}^{\circ}$; Düsseldorf, Universitäts- und Landesbibliothek, D3, fo $3 \mathrm{v}^{\circ}$.

17. Düsseldorf, Universitäts- und Landesbibliothek, $\mathrm{D} 2, \mathrm{f}^{\mathrm{o}} 16 \mathrm{v}^{\circ}$; sur ces trois calendriers, voir K. BODARWÉ, Sanctimoniales litteratae. Schriftlichkeit und Bildung in den ottonischen 
Frauenkommunitäten Gandersheim, Essen und Quedlinburg, Münster, 2004 (Quellen und Studien. Veröffentlichungen des Instituts für kirchengeschichtliche Forschung des Bistums Essen, 10), p. 114, n. 154 ; EAD., "Sanctimoniales litteratae. Schriftlichkeit und Bildung im ottonischen Essen ", dans G. BeRghAus, T. SCHILP et M. SCHLAGHECK dir., Herrschaft, Bildung und Gebet. Gründung und Anfänge des Frauenstifts Essen, 2000, p. 101-117 (avec une illustration du fo $16 v^{\circ}$ du sacramentaire D2) ; H. RöCKELEIN, « Der Kult des heiligen Florinus im Stift Essen », dans J. GERCHOW et T. SCHILP dir., Essen und die sächsischen Frauenstifte im Frühmittelalter, Essen, 2003 (Essener Forschungen zum Frauenstift, 2) ; p. 59-86, spéc. p. $62-65$ (avec une illustration du fo $222 \mathrm{r}^{\circ}$ du sacramentaire D1) et p. 80-84.

18. H. RöCKELEIN, «Leben im Schutz der Heiligen. Reliquientranslationen nach Essen vom 9. bis 11. Jahrhundert », dans G. BERGHAUS, T. SCHILP et M. SCHLAGHECK dir., Herrschaft, Bildung und Gebet..., op. cit., n. 17, p. 87-100.

19. Gesta episcoporum Cameracensium, L. Bethmann éd., op. cit., n. 15, II, 12, p. 459 : «Est autem antiqua relatio quod longo post tempore Saxones, incertum qua causa, ad hanc viciniam devenerunt, et per ipsum vicum transeuntes, audita quidem sacra opinione, partem corporis sacrae virginis asportarunt. »

20. K. KUNZE, "Saturnina », Bibliotheca sanctorum, t. 11, Rome, 1968, col. 669-671; I. GARDILL, Sancta Benedicta. Missionarin, Märtyrerin, Patronin. Der Prachtcodex aus dem Frauenkloster Sainte-Benoîte in Origny, Petersberg, 2005, p.95-97 (sur la contamination des traditions concernant les deux saintes au IX siècle); Hedwig Röckelein a eu la gentillesse de nous communiquer les éléments inédits qu'elle a rassemblés sur Saturnine, nous l'en remercions vivement.

21. On peut aussi se demander si l'épisode de l'enlèvement des reliques par des Saxons n'est pas aussi l'écho déformé de traditions relatives à sainte Maxellende.

22. H. RöCKELEIN, Reliquientranslationen nach Sachsen, op. cit., n. 2, p. 301-302.

23. D. CLAUDE, Geschichte des Erzbistums Magdeburg bis in das 12. Jahrhundert, t. 1, Die Geschichte der Erzbischöfe bis auf Ruotger (1124), Cologne/Vienne, 1972 (Mitteldeutsche Forschungen, 67-1), p. 17-135; on verra aussi désormais les contributions rassemblées dans M. PuHLE dir., Otto der Grosse. Magdeburg und Europa, t. 1, Essays, Mayence, 2001.

24. W. S CHLESINGER, "Zur Geschichte der Magdeburger Königspfalz», Blätter fur deutsche Landesgeschichte, t. 104, 1968, p. 1-31, à la p. 30 ; l'ancrage lotharingien de la politique d'Henri I ${ }^{\text {er }}$ et surtout de son fils Otton $\mathrm{I}^{\text {er }}$ a aussi été mis en évidence par H. BEUMANN, «Die Bedeutung Lotharingiens für die Ottonische Missionspolitik im Osten », Rheinische Vierteljahrsblätter, t. 33, 1969, p. 14-44.

25. D. CLAUDE, Geschichte des Erzbistums Magdeburg, op. cit., n. 23, p. 39-42 ; U. SWINARSKI, Herrschen mit den Heiligen, op.cit., n. 1, p. 149-152 ; d'importants développements ont été très récemment consacrés aux translations de reliques vers Magdebourg par H. RöCKELEIN, « Just de Beauvais alias Justin d'Auxerre : l'art de dédoubler un saint. Avec l'édition de la Passio s. Iustini (BHL 4579) par François Dolbeau et Hedwig Röckelein ", dans M. HEINZELMANN dir., Livrets, collections et textes. Études sur la tradition hagiographique latine, Ostfildern, 2006 (Beihefte der Francia, 63), p. 323-360, aux p. 338-343.

26. Thietmar de Mersebourg († 1018), Chronica, R. Holtzmann éd., MGH, SS rerum Germanicarum, NS, t. 9, Berlin, 1935, II, 17, p. 58.

27. D. A. WARNER, « Henry II at Magdeburg: kingship, ritual and the cult of saints », Early Medieval Europe, t. 3, 1994, p. 135-166.

28. Thietmar De MersebourG, Chronica, op. cit., n. 26, II, 16, p. 56 : «Multa sanctorum corpora imperator ab Italia ad Magadaburg per Dodonem capellanum suimet transmisit »; une recherche fouillée des reliques susceptibles d'avoir été rapportées d'Italie au $\mathrm{x}^{\mathrm{e}}$ siècle a été entreprise par P. Bertrand, en attendant sa publication prochaine, on trouvera à titre indicatif un grand nombre 
de noms de martyrs italiens dans la liste des saints commémorés à Magdebourg au Moyen Âge donnée par B. Schwineköper et G. Wentz (voir n. 5).

29. Un exemple: K. BODARWÉ, « Roman martyrs and their veneration in Ottonian Saxony: the case of the sanctimoniales of Essen », Early Medieval Europe, t. 9, 2000, p. 345-365.

30. P. CORBET, Les saints ottoniens. Sainteté dynastique, sainteté royale et sainteté féminine autour de l'an Mil, Sigmaringen, 1986 (Beihefte der Francia, 15).

31. Sur tout ceci, voir R. E. BARTH, Der Herzog in Lotharingien im 10. Jahrhundert, Sigmaringen, 1990.

32. H. SPRÖMBERG ; «Die lothringische Politik Ottos des Grossen », Rheinische Vierteljahrsblätter, 11, 1941, p.1-101; reimp.dans ID., Beiträge zur belgisch-niederländischen Geschichte, Berlin, 1959, p. 111-223 (ici spéc. 134-150).

33. Sur le rôle de Fulbert à Ingelheim, voir C. MÉRIAUX, « Fulbert », op. cit., n. 11, p. 529-532.

34. Flodoard, Historia Remensis ecclesiae, éd. M. StRATMANN, MGH, SS, t. 36, Hanovre, 1998, I, 4, p. 71: « Beati denique Timothei ossa rex Otto concedente Artaldo archiepiscopo transferri fecit in Saxoniam et monasterium monachorum in eius instituit honore ».

35. J. DEVISSE, Hincmar, archevêque de Reims (845-882), Genève, 1975-1976 (Travaux d'histoire éthico-politique, 29), t. 1, p. 69, n. 203 qui commente une lettre d'Hincmar à Folcuin, évêque de Thérouanne.

36. Vita Autberti, J. GHESQUiÈRE éd., op. cit., n. 9, IV, 31, p. 563 : « sancti confessores [...] per quos Cameracensis civitas felix in propagine, fertilis in germine, et temporalem meruerat salutem et supernam sperabat beatitudinem. »

37. Ibid., IV, 32, p. 563 : «scilicet ut Cameracensis civitas quae confinium imperii ejus a Francis disterminat, et Magadaburc, quae alio confinio sub regno ejus Sclavos a Germania eliminat, beati viri munitae presidio, tanquam forti circumdatae muro tuerentur. »

\section{RÉSUMÉS}

La documentation narrative, diplomatique et liturgique permet de mettre en évidence l'existence de plusieurs translations de reliques de Cambrai et de ses environs vers la Saxe à la fin des années 940 ; elles concernent les saints Aubert, Géry, Madelberte, Maxellende et peut-être aussi une énigmatique sainte Saturnine. Contrairement aux translations de reliques de l'époque carolingienne qui manifestaient l'intégration de la Saxe dans l'empire franc, les translations du $\mathrm{x}^{\mathrm{e}}$ siècle sont révélatrices d'un mouvement inverse: l'ancrage très net de la Lotharingie dans l'espace politique ottonien.

The narrative, diplomatic and liturgical documentation shows several transfers of relics from Cambrai and its neighbourhood towards Saxony at the end of the 940's; they concern in particular the saints Aubert, Géry, Madelberte, Maxellende and perhaps also the enigmatic saint Saturnine. Contrary to the transfer of relics during the Carolingian period that showed the integration of Saxony into the Frankish empire, the transfers of the xth century reveal an opposite movement: the marked anchorage of Lotharingia in the Ottonian political region. 
INDEX

Mots-clés : translations de reliques, Lotharingie, Cambrai, Saxe, Otton Ier

Keywords : relics transfer, Lotharingia, Cambrai, Saxony, Otto I

\section{AUTEURS}

\section{PAUL BERTRAND}

CNRS, UPR 841, Institut de recherche et d'histoire des textes, section diplomatique, Centre Augustin-Thierry, 3B, avenue de la Recherche scientifique, 45071 Orléans cedex 2

\section{CHARLES MÉRIAUX}

Université Lille III-Charles-de-Gaulle - UMR 8529 IRHIS, BP 60149, 59563 Villeneuve-d'Ascq cedex 